\title{
MCT1 and MCT4 Expression During Myocardial Ischemic-Reperfusion Injury in the Isolated Rat Heart
}

\author{
Yi Zhu Jing Wu Shi-Ying Yuan
}

Department of Anesthesiology and Intensive Care Medicine, Union Hospital, Tongji Medical College, Huazhong University of Science and Technology, Wuhan

\section{Key Words}

Lactate $\cdot$ Myocardial ischemia-reperfusion injury $•$ Monocarboxylate transporters

\begin{abstract}
Background/Aims: Myocardium ischemia-reperfusion (I/R) injury can be caused by imbalances in cellular metabolism. Lactate, transported by monocarboxylate transporters (MCTs), has been implicated as a mechanism in this process. The present study was designed to investigate the expression and functional role of MCTs in rat hearts during ischemia and reperfusion. Methods: Langendorff-perfused rat hearts were subjected to 20 minutes stabilization, 30 minutes of global ischemia and 60 minutes reperfusion. Hearts were collected serially for detecting expression changes in MCT1, MCT4 during myocardial I/R injury and lactate concentration was measured. Post-ischemic left ventricular function and infract size were determined at end-point, followed by the pretreatment of D-lactate, a competitive inhibitor of MCTs. Results: MCT4 was significantly increased following global ischemia and MCT1 expression was increased during the early stages of reperfusion in isolated rat hearts, while the expression of the ancillary protein CD147 was increased during I/R injury. We determined increases in AMPK phosphorylation status, which was significantly elevated following ischemia and early reperfusion. Blocking monocarboxylate transport by competitive inhibition with D-lactate caused decreased left ventricular performance and increased infarct size. Conclusion: Increased MCT4 expression facilitates lactate extrusion during the ischemic period, while increased MCT1 may facilitate lactate transport into and out of cells simultaneously during early reperfusion, with increases in AMPK phosphorylation status during the myocardial I/R period. Lactate transport by MCTs has a profound protective effect during myocardial ischemia reperfusion injury.
\end{abstract}

Jing Wu

and Shi-Ying Yuan
Department of Anesthesiology and Intensive Care Medicine, Union Hospital Tongji Medical College, Huazhong University of Science and Technology, 1277 Jiefang Avenue Wuhan 430022 (China)

E-Mail wujingjingwu@Gmail.com or E-Mail yuanshiyingyingshiyuan@Gmail.com 


\section{Introduction}

Ischemic heart disease, which is caused by an interruption in blood flow to the myocardium, is predicted to become the leading cause of mortality globally by the year 2020 [1]. Emergent reperfusion therapy for the restoration of coronary patency is currently the main treatment. However, immediate reperfusion has been shown to accelerate cell death and increase the extent of infarction, which is undesirable but inevitable. This process is known as myocardial ischemic-reperfusion (I/R) injury [2]. Research to protect the heart against myocardial I/R injury has progressed for decades, both in clinical practice and basic research. Nowadays, many studies are focused on investigating the underlying intracellular mechanisms and signal transduction complexes involved in cardioprotection. Immediate recanalization remains the gold standard for current clinical treatments, although there are known consequences of reperfusion injury. There remains an increasing need for novel therapeutic strategies for cardioprotection against I/R injury.

Myocardial I/R injury is a metabolic event caused by a short supply of energy. Alterations in energy metabolism is a vital issue triggering myocardial I/R injury [3]. Following the onset of ischemia, oxidative phosphorylation of glucose is inhibited and glycolysis is the only mechanism of ATP production [3]. During the early stages of reperfusion, glycolysis continues while glucose oxidation is inhibited [4]. Lactate, which links glycolysis and oxidative metabolism [5], may participate in the imbalance of energy metabolism during I/R injury.

Lactate, the end product of glycolysis resulting from hypoxia, once was treated as a metabolic waste product. However, the potential therapeutic targets of lactate are now being recognized. Lactate plays a central role in cellular metabolism and metabolic communication between tissues [6]. Transport of lactate into and out of cells is facilitated by a family of monocarboxylate transporters (MCTs) [7]. Among the isoforms of MCT, MCT1 and MCT4 are considered to be the key transporters in cardiac muscle [8]. The transportation function of these isoforms is associated with CD147 (EMMPRIN) [9], a key glycoprotein, which assists MCT1 and MCT4 in folding, stability, membrane expression, and functionality. Moreover, lactate dehydrogenase (LDH) is involved in the shuttling of lactate between cells via the cellcell lactate shuttle [10].

Recent reports have shown that AMP-activated protein kinase (AMPK) activation increases MCT1 and MCT4 protein expression in skeletal muscle [11-13]. Furthermore, the current view is that AMPK protects the heart from I-R injury by sustaining an energy supply $[2,14]$. It therefore follows that there may be an analogous correlation between MCTs and AMPK in cardiac muscle.

The purpose of the present study was two-fold: 1. Identify MCT1 and MCT4 expression in isolated rat hearts during acute I/R injury; and 2. Investigate the functional role of MCTs in acute I/R injury.

\section{Materials and Methods}

Adult male Sprague-Dawley rats (250-300g) were purchased from the Hunan Weasleyg Scene of Experimental Animals Co, LTD (Hunan China). All animal protocols were performed in accordance with the U.S. National Institute of Health guidelines (NIH Publication No.85-23, revised 1996) and approved by the Animal Care and Use Committee of Tongji Medical College of Huazhong University of Science and Technology.

Isolated heart perfusion and experimental groups

Rats were anesthetized with sodium pentobarbital (50 mg/kg, i.p.) and injected with heparin (500 IU i.p.). Hearts were rapidly excised and mounted on a Langendorff apparatus (ADInstruments, Australia) and perfused with Krebs-Henseleit buffer (KHB; containing $120.0 \mathrm{mM} \mathrm{NaCl}, 4.5 \mathrm{mM} \mathrm{KCl}, 20.0 \mathrm{mM} \mathrm{NaHCO}{ }_{3}$,

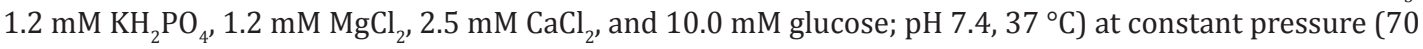
$\mathrm{mmHg}$ ), which was previously equilibrated with $95 \% \mathrm{O}_{2}-5 \% \mathrm{CO}_{2}$. Hearts were randomly divided into two 


\begin{tabular}{|c|c|c|}
\hline Cellular Phys & Cell Physiol Biochem 2013;32:663-674 & \\
\hline and Biochemistry & $\begin{array}{l}\text { DOI: } 10.1159 / 000354470 \\
\text { Published online: September 10, } 2013\end{array}$ & $\begin{array}{l}\text { O } 2013 \text { S. Karger AG, Basel } \\
\text { www.karger.com/cpb }\end{array}$ \\
\hline
\end{tabular}

series. Series 1: hearts were perfused with a 20 minute stabilization period $(\mathrm{T} 0, \mathrm{n}=8)$, or perfused with 20 minutes stabilization, 30 minutes of global ischemia ( $(30, n=8)$, or reperfused for 15 minutes following ischemia (R15, n=8), or reperfused for 60 minutes following ischemia (R60, n=8). These hearts were collected to determine the expression and level of MCT1, MCT4, CD147, LDH and AMPK phosphorylation. Coronary effluent at the end of the study in each group was collected for lactate release measurements. Once series 1 was accomplished, series 2 was performed to study lactate transport during the I/R period mediated by MCTs and to test whether lactate itself influences heart performance. Series 2: hearts were perfused with 20 minutes stabilization, 30 minutes global ischemia, and 60 minutes reperfusion $(I / R, n=9)$, and compared with hearts perfused for 5 minutes with $100 \mathrm{mM}$ of either D-lactate (D-LAC, $\mathrm{n}=9$ ) or L-lactate (L-LAC, $\mathrm{n}=9$ ) prior to ischemia. During perfusion hemodynamic parameters, including left ventricular (LV) function, were recorded continuously. At the end of perfusion, besides the measurements determined in series 1 , hearts were collected for the determination of myocardial apoptosis and necrosis, and myocardial infarct size.

Real-time RT-PCR

MCT1 and MCT4 mRNA levels in rat cardiomyocytes were determined using quantitative real-time PCR analyses. Myocardium samples were homogenized with Trizol reagent (Invitrogen, USA) and total RNA was extracted according to the manufacturer's protocol. Concentration and purity of total RNA was determined using a BioPhotometer (Eppendorf, Germany). Total RNA was then reverse-transcribed into complementary DNA (cDNA) using a cDNA synthesis kit (TOYOBO, Japan) which was performed according to the manufacturer's protocol. Real-time PCR was performed using a StepOne Real-Time PCR System (Applied Biosystems, USA) and Thunderbird SYBR qPCR Mix (TOYOBO, Japan). The RT-PCR conditions were $42^{\circ} \mathrm{C} / 30 \mathrm{~min}$, and $80^{\circ} \mathrm{C} / 5 \mathrm{~min}$ for reverse transcription. PCR involved pre-denaturing at $95^{\circ} \mathrm{C}$ for 1 minute, then 40 cycles of $95^{\circ} \mathrm{C}$ for 15 seconds, $58^{\circ} \mathrm{C}$ for 20 seconds, and $72^{\circ} \mathrm{C}$ for 20 seconds. $\beta$-actin was used as the housekeeping gene. Levels of MCT1 and MCT4 mRNA were calculated based on the $2^{-\Delta \Delta \mathrm{Ct}}$ comparative quantification method. The PCR primers used included: MCT1 forward primer (5'-CTC TGG GCG CCG CGA GAT AC-3'), reverse primer (5'- CAA CTA CCA CCG CCC AGC CC-3'); MCT4 forward primer (5'- CCA GGC CCA CGG CAG GTT TC-3'), reverse primer (5'- GCC ACC GTA GTC ACT GGC CG-3); $\beta$-actin forward primer (5'-ACG TTG ACA TCC GTA AAG AC-3'), reverse primer (5'-GAA GGT GGA CAG TGA GGC-3'). All samples for each gene were run in duplicate.

\section{Western blotting}

$\mathrm{LV}$ tissue was homogenized and protein concentration of the supernatant was determined using a BCA method (Pierce, USA). The supernatant containing $50 \mu \mathrm{g}$ of protein was separated using a $10 \%$ SDSPAGE and transferred onto polyvinylidene difluoride membranes (Millipore, USA) by electrophoresis. The membranes were then blocked in TBS containing $0.1 \%$ Tween- 20 and $5 \%$ nonfat milk at room temperature for 1 hour. The membranes were incubated in primary antibodies (anti-Actin, anti-MCT1, anti-MCT4 and anti-CD147, 1:250, Santa Cruz, USA; anti-AMPK- $\alpha$, anti-phospho-AMPK- $\alpha, 1: 1000$, Cell Signalling Technology, USA) at $4{ }^{\circ} \mathrm{C}$ overnight, followed by a HRP-conjugated anti-rabbit IgG secondary antibody or anti-mouse IgG secondary antibody (1:5000, Proteintech,China). Target proteins were detected using the ChemiDocXRS+ chemiluminescence imaging system (Bio-Rad, USA). Protein bands were quantified by Image Lab image acquisition and analysis software (Bio-Rad, USA).

\section{Measurement of myocardial LDH content and activity}

Cardiac tissues were homogenized in PBS (pH 7.4) and centrifuged for extracting supernatant. Myocardial LDH levels were measured using a rat LDH enzyme-linked immune sorbent assay (ELISA) kit (IBL, Germany). Myocardial LDH activities were measured using LDH kits (Nanjing Jiancheng Biotech, China).

\section{Lactate concentration in coronary effluent}

Lactate concentration in coronary effluent was measured using a lactic acid kit (Nanjing Jiancheng Biotech, China) according to the manufacturer's instructions. 


\section{Cardiac function measurements}

Cardiac LV function was recorded continuously via a water-filled balloon placed into the left ventricle and attached to a pressure transducer after the heart was mounted on a Langendorff apparatus (ADInstruments, Australia). Cardiac functional assessment was then collected and analyzed using a PowerLab data-acquisition system with LabChart software (version 7 for Windows, ADInstruments, Australia). Measured parameters included LV developed pressure (LVDP), LV end-diastolic pressure (LVEDP), and maximum LVDP increase $(+\mathrm{dp} / \mathrm{dt})$ and decrease $(-\mathrm{dp} / \mathrm{dt})$ rates. Coronary flow rate $(\mathrm{CF})$ was calculated from the coronary effluent.

\section{Myocardial infarct size assessment}

At the end of the experiment, the hearts were frozen at $-20^{\circ} \mathrm{C}$ for 2 hours and then sliced transversely into 2-mm-thick sections and incubated in a $1 \%$ solution of 2,3,5-triphenyltetrazoliumchloride (TTC; Amresco, USA) for 15 minutes at $37^{\circ} \mathrm{C}$, followed by transfer into a $4 \%$ paraformaldehyde-phosphatebuffered saline solution for fixation at $4^{\circ} \mathrm{C}$. The infarcted tissue remained unstained (white), whereas normal tissue was stained red. Infarct area was traced and the total area was calculated using Image J software (NIH, USA). Infarct size was expressed as a percentage of total ventricular area.

\section{Determination of myocardial apoptosis and necrosis}

Terminal dUTP nick-labelling (TUNEL) was assessed using a kit (Roche, Switzerland) according to the manufacturer's instructions in order to determine cardiomyocyte apoptosis. TUNEL-positive nuclei with chromatin condensation and fragmented nuclei were considered as probable apoptotic cells. Quantification of apoptosis was determined by counting the TUNEL-positive myocyte nuclei from three random fields per section using light microscopy at x200 magnification by an investigator who was blinded to the study. LV tissues were fixed in $4 \%$ paraformaldehyde and embedded in paraffin. Sections were stained using haematoxylin and eosin (HE), and then histologically assessed by a pathologist for acute myocardial necrosis, interstitial oedema, and cellular swelling.

\section{Statistics}

All values are shown as mean \pm standard error of the mean (SEM). GraphPad Prism (version 5 for Windows, GraphPad software, USA) was used for all statistical analyses. Differences in the recovery of postischemic hemodynamic parameters were tested using a two-way analysis of variance (ANOVA) followed by a Bonferroni's post-hoc test for multiple comparisons. Other data were subjected to one-way ANOVA followed by a Tukey's post-hoc test. Statistical significance was set at $\mathrm{P}<0.05$.

\section{Results}

MCT1 and MCT4 mRNA expression in cardiomyocytes

Series 1: Compared with the level prior to ischemia, MCT1 mRNA was significantly elevated following 15 minutes reperfusion ( $\mathrm{P}<0.01$; Fig. $1 \mathrm{~A})$, while MCT4 mRNA was rapidly increased after 30 minutes ischemia $(\mathrm{P}<0.001$; Fig. $1 \mathrm{~B})$. There was no obvious difference when the rest group was compared to T0. Series 2: compared with the I/R group, MCT1 and MCT4 mRNA was increased in the D-LAC group ( $\mathrm{P}<0.01$, respectively, Fig. $1 \mathrm{C}$, D), while the L-LAC and I/R groups were similar.

\section{Western blotting}

Series 1: Western blotting showed that MCT4 protein expression was significantly increased following 30 minutes ischemia $(\mathrm{P}<0.01$; Fig. 2B). After 15 minutes reperfusion, protein expression decreased to the level occurring before ischemia until 60 minutes reperfusion. MCT1 expression was markedly increased after 15 minutes reperfusion $(\mathrm{P}<0.01$; Fig. 2A) and the level was normalized after 60 minutes reperfusion. CD147 protein expression was increased during the I/R period compared to the level before ischemia $(\mathrm{P}<0.05$; Fig. 2C). AMPK phosphorylation status [phospho-AMPK (Thr172)/total AMPK] was significantly elevated following 30 minutes ischemia and 15 minutes reperfusion $(\mathrm{P}<0.01$, $\mathrm{P}<0.05$, respectively, Fig. 2D). After 60 minutes reperfusion, the level was normalized. Series 


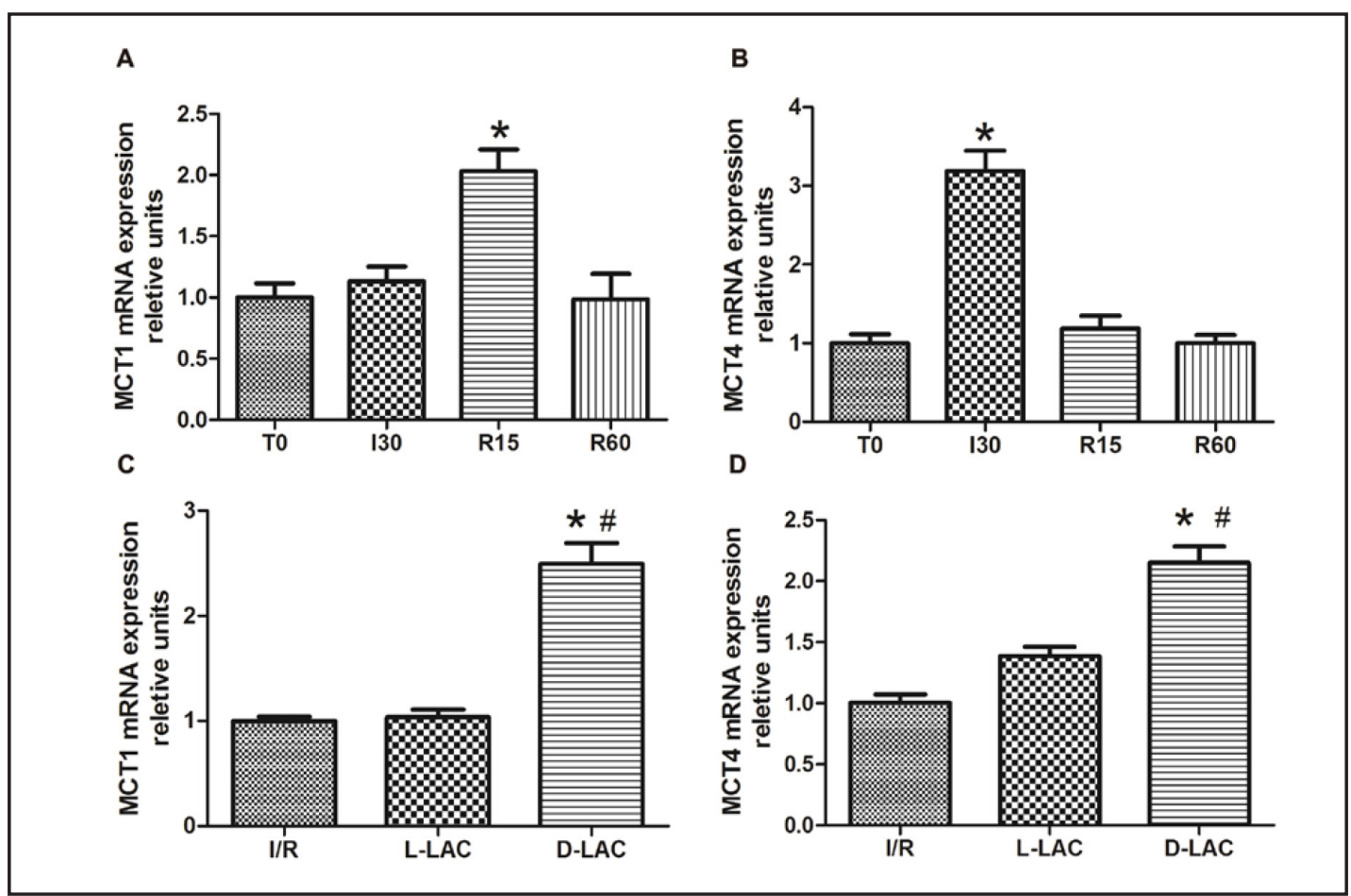

Fig. 1. (A and B) Expression of MCT1 and MCT4 mRNA during myocardial I/R period. Values presented are means \pm SEM. ${ }^{*} \mathrm{P}<0.05$ vs T0 group. ( $C$ and D) Expression of MCT1 and MCT4 mRNA in hearts perfused with L- and D- lactate or without lactate. Values are expressed as means \pm SEM. ${ }^{*} \mathrm{P}<0.05$ vs I/R group, \# $\mathrm{P}<0.05$ vs L-LAC group.

2: There were no statistically significant differences between groups with regard to MCT1, MCT4 and CD147 protein expression (Fig. 2E, F, G). However, AMPK phosphorylation status was significantly elevated in the D-LAC group compared with both L-LAC and I/R groups $(\mathrm{P}<0.01$, respectively, Fig. $2 \mathrm{G})$, while no statistical differences were observed between the L-LAC and I/R groups.

\section{LDH content and activity in coronary effluent}

Series 1: LDH content was elevated after 30 minutes ischemia and 15 minutes reperfusion $(\mathrm{P}<0.01, \mathrm{P}<0.05$, respectively, Fig. 3A). After 60 minutes reperfusion, the level dropped to that observed before ischemia. The activities of LDH were in parallel to the content during the I/R period $(\mathrm{P}<0.05, \mathrm{P}<0.05$, respectively, Fig. $3 \mathrm{~B})$. Series 2 : There were no statistically significant differences between groups (Fig. 3C, D).

\section{Lactate concentration}

Series 1: Lactate concentration was elevated after 30 minutes ischemia $(\mathrm{P}<0.01$; Fig. 4 ). The concentration rapidly fell to the level before ischemia after 15 minutes reperfusion. Series 2: There were no statistically significant differences between groups (Fig. 4C, D).

\section{Cardiac LV function}

Following sustained ischemia, hearts pretreated with D-lactate had a worse performance in the recovery of post-ischemic hemodynamic parameters. Compared with hearts pretreated with L-lactate or not pretreated, LVDP, $\pm \mathrm{dp} / \mathrm{dt}$ were significantly depressed in D-lactate $(\mathrm{P}<0.01, \mathrm{P}<0.01$, respectively, Fig. 5A, B, C). LVEDP ascended during reperfusion in D-lactate compared with L-lactate or I/R, respectively $(\mathrm{P}<0.01, \mathrm{P}<0.01$, respectively, Fig. 5D). CF of hearts pretreated with $\mathrm{D}$-lactate was also reduced compared with L-lactate or I/R $(\mathrm{P}<0.01$, 


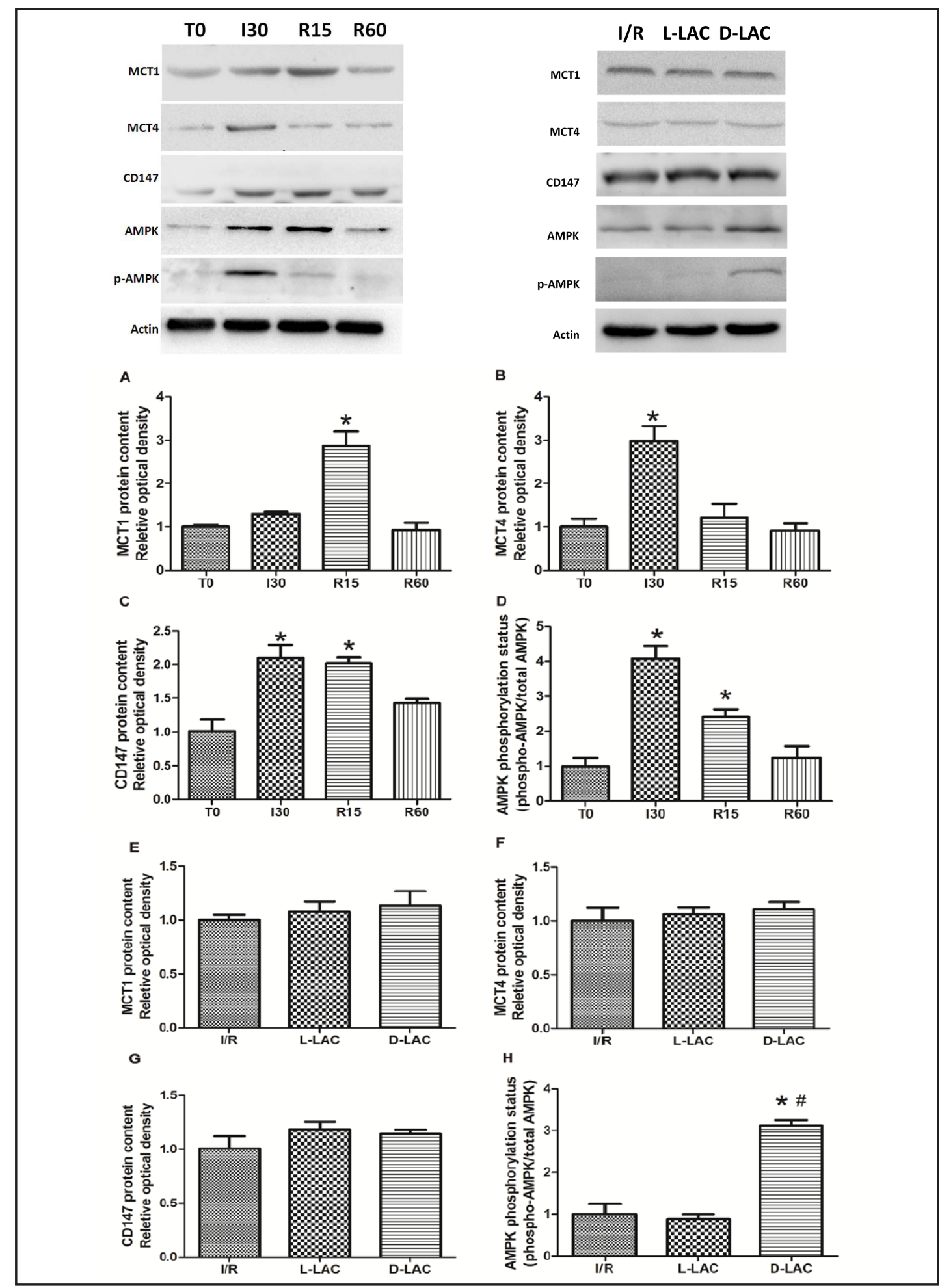

Fig. 2. (A-D) Western blot analyses of MCT1 and MCT4, CD147 expression and AMPK phosphpryation status during myocardial I/R period. Actin was used as an internal reference.Values are expressed as means \pm SEM.*P $<0.05$ vs T0 group. (E-H) Western blot analyses of MCT1 and MCT4, CD147 expression and AMPK phosphpryation status in hearts perfused with L- and D-lactate for 5 minutes or without lactate before 30 minutes of global ischemia and 60 minutes reperfusion. Values are expressed as means \pm SEM. ${ }^{*} \mathrm{P}<0.05$ vs I/R group, \# P <0.05 vs L-LAC group. 


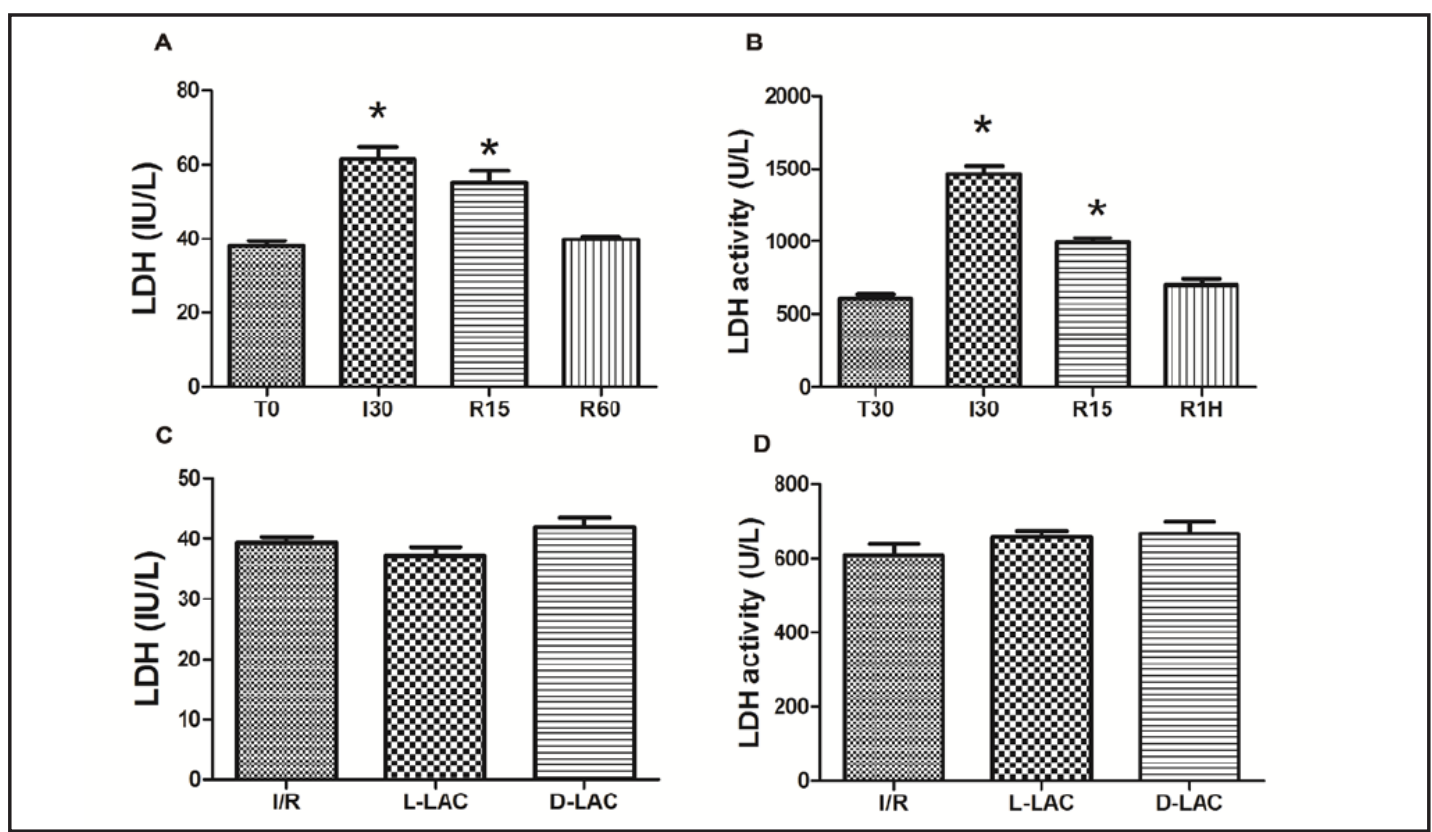

Fig. 3. (A and B) LDH content and activity in cardiac tissue during myocardial I/R period. Values are expressed as means \pm SEM. ${ }^{*} \mathrm{P}<0.05$ vs T0 group. (C and D) LDH content and activity in cardiac tissue perfused with D-lactate and L-lactate or without lactate. Values are expressed as means \pm SEM.

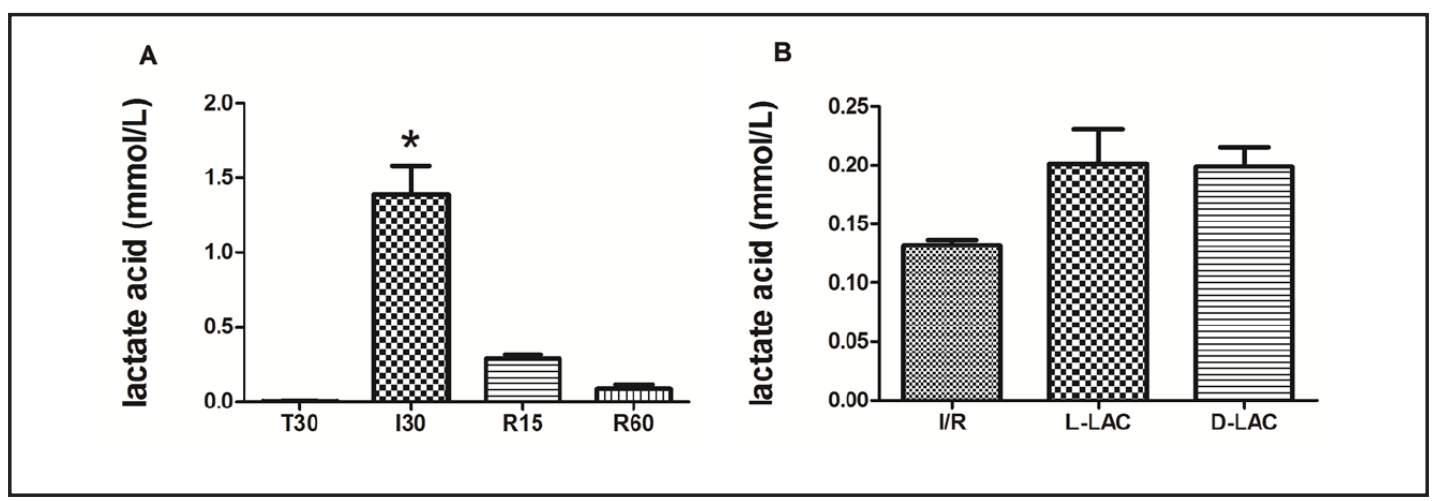

Fig. 4. (A) Concentration of lactate in the coronary effluent during the myocardial I/R period. Values are expressed as means \pm SEM. $* \mathrm{P}<0.05$ vs T0 group. (B) Concentration of lactate in the coronary effluent perfused with D-lactate and L-lactate or without lactate. Values are expressed as means \pm SEM.

$\mathrm{P}<0.05$, respectively, Fig. 5E). There were no differences between groups L-lactate and I/R during reperfusion in these parameters except $\pm \mathrm{dp} / \mathrm{dt}$ at the early stage of reperfusion $(\mathrm{P}<0.05$, Fig. 5B, C).

\section{Infarct size}

Infarct size in each group was evaluated after 60 minutes reperfusion. D-lactate significantly increased the infarct size percentage compared with L-lactate and I/R $(\mathrm{P}<0.05$, $\mathrm{P}<0.05$, respectively, Fig. 6). There were no differences between L-lactate and I/R groups.

\section{Myocardial apoptosis and necrosis}

Using the TUNEL assay, a large number of TUNEL-positive cells were observed in hearts pretreated with D-lactate, while few TUNEL-positive cells were visible in the L-lactate and 
A

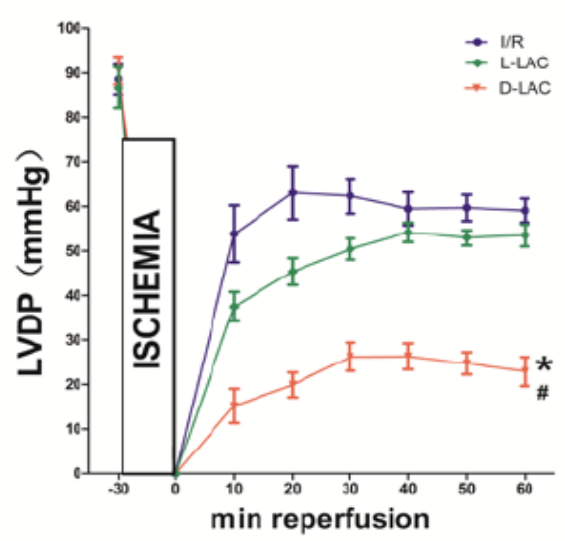

c

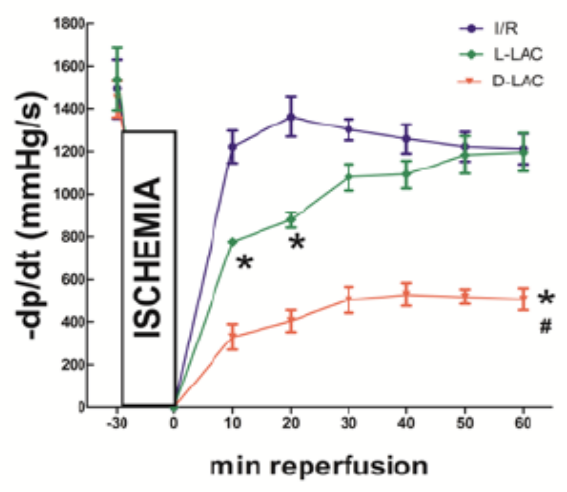

E

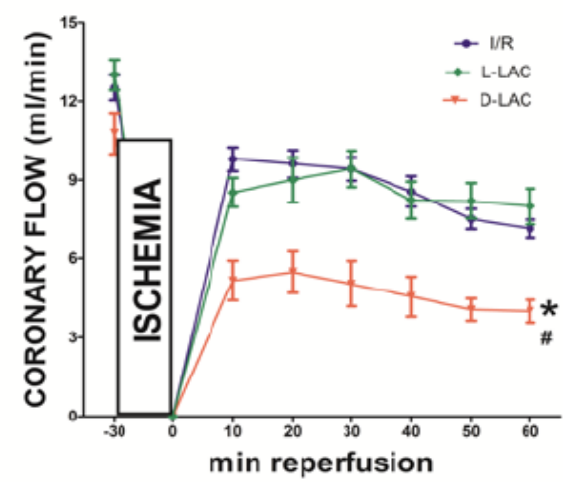

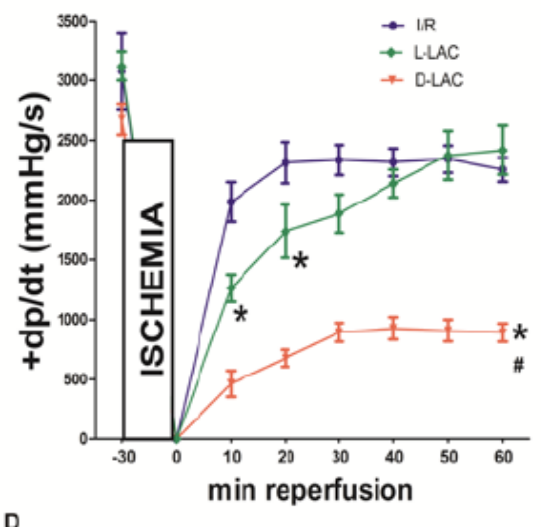

D

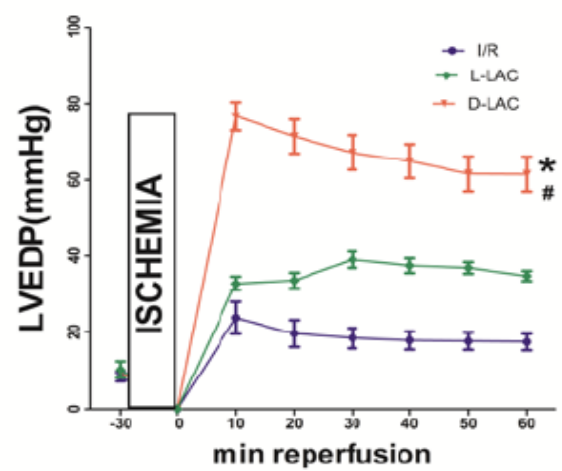

Fig. 5. Rat hearts were perfused with D-lactate (D-LAC, $n=9$ ) and L-lactate (L-LAC, $n=9$ ) for 5 minutes before 30 minutes of induced global ischemia and 60 minutes reperfusion, and compared with global ischemia without lactate (I/R, n=9). (A-E) Recovery of left ventricular developed (LVDP) and end-diastolic pressures (LVEDP), coronary flow (CF), and maximum LVDP increase or decrease of left ventricular pressure $( \pm \mathrm{dP} /$ $\mathrm{dt})$ are shown (mean \pm SEM). -30 and 0 indicates pre-ischemic values, the rest are minutes of reperfusion. Hearts perfused with D-LAC had worse performance than the other groups. Values are expressed as means \pm SEM. $* \mathrm{P}<0.05$ vs I/R group, \# $\mathrm{P}<0.05$ vs L-LAC group.

I/R groups. The percentage of apoptotic cells in D-lactate were significantly increased compared with the L-lactate or I/R groups $(\mathrm{P}<0.01, \mathrm{P}<0.01$, respectively, Fig. 7). There was no difference between the L-lactate and I/R groups. D-lactate increased the extent of cellular swelling and extracellular matrix oedema (Fig. 8). 
Fig. 6. D-lactate increases the myocardial infarct size. Values are expressed as means \pm SEM. ${ }^{*} \mathrm{P}<0.05$ vs I/R group, \# P <0.05 vs L-LAC group.

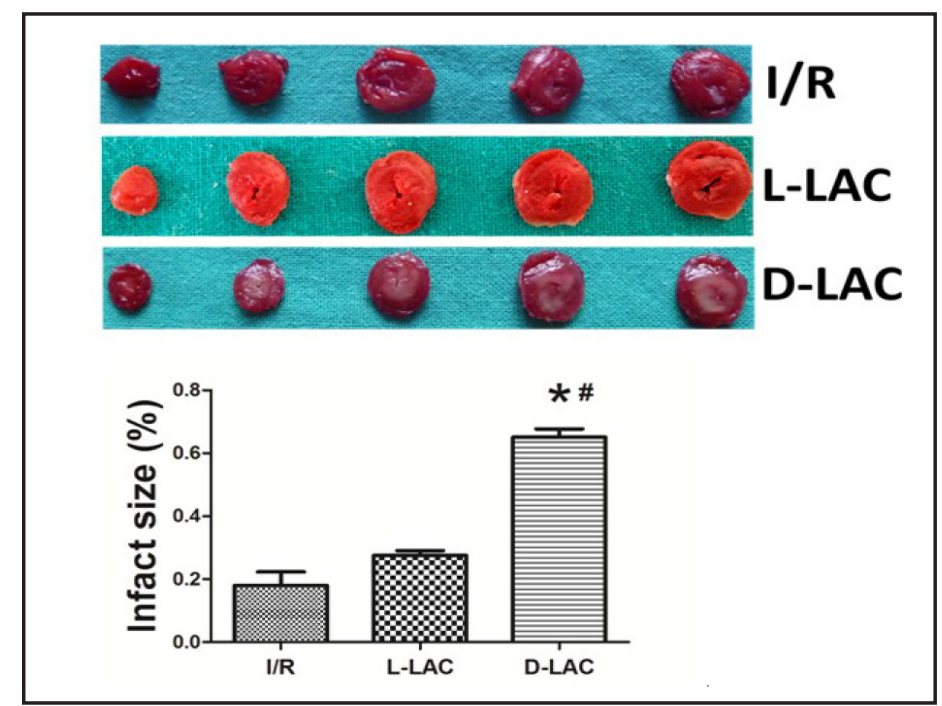

Fig. 7. TUNEL assay. D-lactate increases apoptosis of cardiomyocytes. Values are expressed as means \pm SEM. ${ }^{*} \mathrm{P}<0.05$ vs $\mathrm{I} / \mathrm{R}$ group, \# $\mathrm{P}<0.05$ vs L-LAC group. Original magnification $\times 400$.
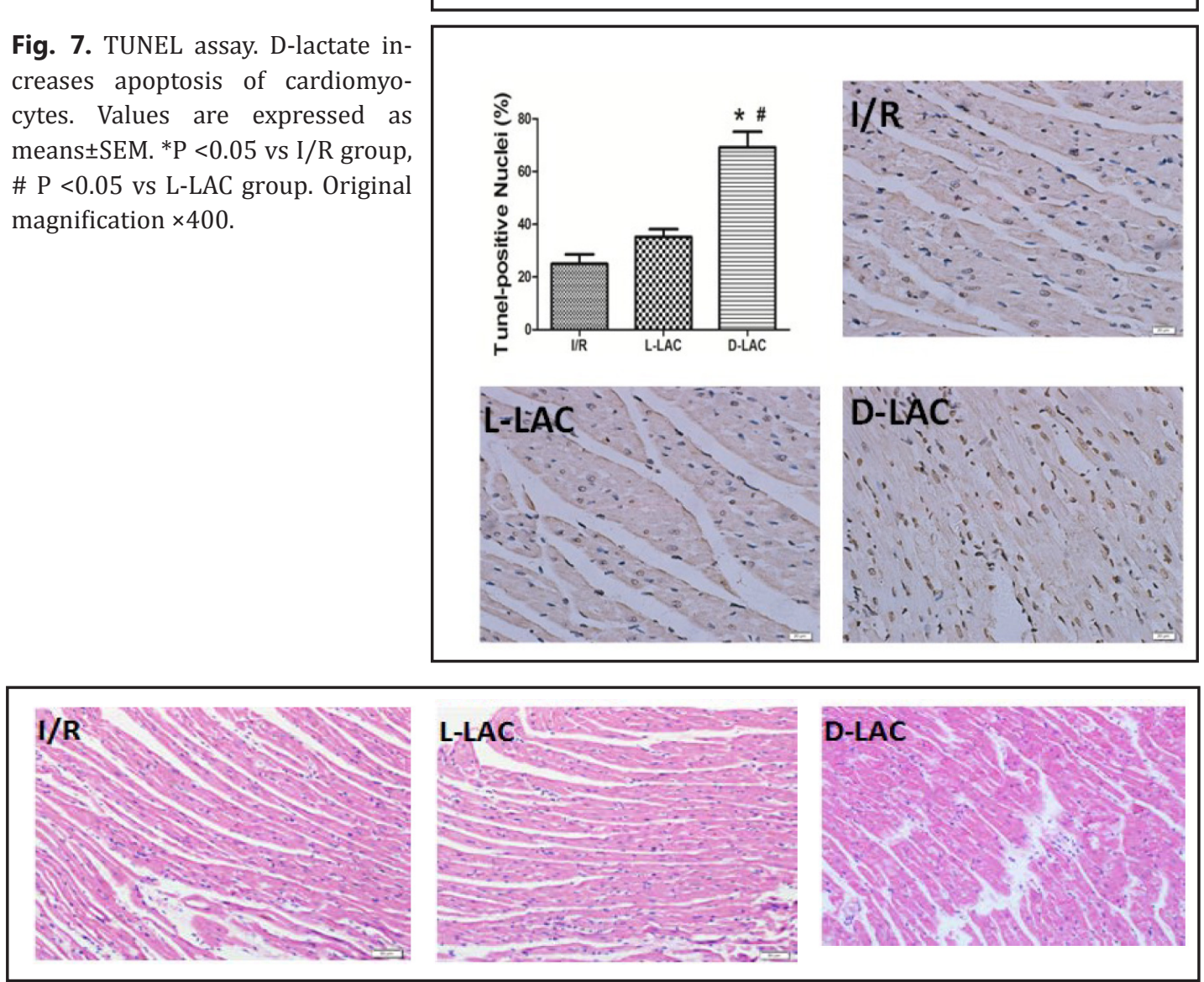

Fig. 8. Representative photomicrographs of HE-stained left ventricular tissue sections. Original magnification $\times 200$.

\section{Discussion}

Under physiological conditions, myocardial ATP production is dependent chiefly on oxidative decarboxylation of fatty acids, providing $60-80 \%$ of the energy demand. The remaining ATP requirement is supplied by glucose and lactate in nearly equal proportions [15], and other substrates such as amino acids and ketone bodies. Co-utilization is determined 
by both the amount of substrate energy generated in the circulation and complex intracellular regulatory mechanisms [16]. However, this regulated balance is altered significantly during the myocardial I/R period. ATP generated from glycolysis prioritizes the function of the ion pump in the cell membrane, while ATP from glucose oxidation promotes the recovery of normal contractile function in the post-ischemic heart [17, 18]. Therefore, reductions in ATP generation during the I/R period results in the accumulation of intracellular ions and myocardial dysfunction. Ischemic-reperfusion injury then occurs. As mentioned above, lactate is not just the production of glycolysis, but the energy substrate for glucose oxidation via the Krebs cycle. Since the heart is an efficient lactate consumer and producer [19], we speculate that lactate transport is associated with energy imbalance during the myocardial I/R period.

Alterations in the expression of the lactate shuttle machinery may affect cellular transport and oxidation of lactate [20]. In earlier studies of lactate transport in the heart in relation to myocardial ischemia [21], Halestrap et al. assumed that there are two MCT isoforms in the heart, one being MCT1 which is found to be expressed in almost all tissues [7], while the other is MCT4, or at least this has been argued [8, 22]. Halestrap believed MCT4 is widely expressed and especially restricted in glycolytic tissues while MCT4 is absent in the heart. However, hypoxia-induced MCT4 expression has been reported by McClelland and Brooks [20]. In addition, another study demonstrated that MCT4 is rapidly induced by hypoxia through the HIF-1 $\alpha$ pathway [23]. MCT4 is adapted to export lactate, although it has low affinity for L-lactate because of its very low affinity for pyruvate $(\mathrm{Km}, 150 \mathrm{mM})$ while other isoforms are extremely high, which would be of particular importance since it prevents the loss of pyruvate from the cell. This is essential because high rates of glycolysis requires that NADH produced in glycolysis is re-oxidised, which is achieved by pyruvate being converted to lactate [24]. In a global Langendorff rat heart model, glycolysis is the only means for energy generation. During global ischemia, it slows gradually at 30 minutes of ischemia, and may continue even longer until lower $\mathrm{pH}$ mediates inhibition of glycolysis before complete glycogen depletion $[25,26]$. In humans, myocardial ischemia is a common occurrence which extends from hours to days preceding death. Therefore, the hypothesis that MCT4 is totally absent from the heart is unsound. In our study, MCT4 was markedly expressed following 30 minutes ischemia with lactate concentrations in the coronary effluent very high. Moreover, expression of LDH and CD147 is also increased following ischemia. This result suggests that it is MCT4 which mainly facilities lactate extrusion during the ischemia period.

After 15 minutes reperfusion, we found that MCT1 is significantly increased in the isolated heart. This result is consistent with Martinov's study in mice [27]. The predominant role of MCT1 is to facilitate unidirectional proton-linked transport of L-lactate across the plasma membrane. Intracellular and extracellular $\mathrm{pH}$ achieved 5.9 and 5.5, respectively after 20 minutes ischemia [26], MCT1 may facilitate lactate extrusion during the ischemia period and the onset of reperfusion to maintain non-acidity in the intracellular environment. However, intracellular $\mathrm{pH}$ in cardiomyocytes returns to normal within a few minutes of reperfusion [26], and our results showed that after 15 minutes reperfusion, lactate concentration in the effluent nearly drops to the level observed before ischemia. In addition, LDH and CD147 are also active at this time, which indicates that lactate metabolism is still active. Therefore, it seems that the function of the abundant expression of MCT1 at this moment is in question. During the early stages of reperfusion, glycolysis may exceed regional imbalance between glycolysis and glucose oxidation $[4,28,29]$. As lactate is a preferred fuel for the injured heart [30], and it is proven to provide simultaneous lactate uptake and extrusion as functionally separate metabolic pathways within the heart [31], we speculate that MCT1 affords lactate transport into and out of the cells simultaneously at this moment. This requires further study.

Considering the lack of common inhibitors of both MCT1 and MCT4 [22], D-lactate is a valuable tool with which to investigate L-lactate transport [32]. When MCTs were competitively blocked using D-lactate, left ventricular function was depressed and infarct size increased. This indicates that lactate transport by MCTs has a profound protective effect on myocardial ischemia reperfusion injury. Although myocardial MCT1 and MCT4 expression 
were not influenced by either L- nor D-lactate in this study, we found that their mRNA expression and AMPK phosphorylation status increased following D-lactate pretreatment. Considering lactate may be a major energy resource for the failing heart, and may preserve or even improve myocardial performance [30,33], this possibly indicates that the hearts are failing and have a greater reliance on carbohydrates. It is suggested that regulation of MCT expression seems to be translational [13]. We did not find increased MCT expression, which may be due to the time frame of observation.

AMPK has been proposed as a putative target to limit the extent of I/R injury [14]. It protects the heart from I/R injury by sustaining energy supply during ischemic stress and reducing the likelihood of cardiomyocyte cell death. Furthermore, in other studies, AMPK activation induced increases in MCT1 and MCT4 protein content in rat skeletal muscle [11-13]. In the current study, we also found that MCT1 and MCT4 protein expression was increased with increases in AMPK phosphorylation status during the I/R period. As a master metabolic regulator in mammalian cells, AMPK activity may be important and required for up-regulating MCT1 and MCT4 expression, to facilitate lactate uptake and release in the heart for meeting cellular homeostasis and energy demands during the myocardial I/R period. The specific mechanism needs further study.

There are several limitations in our study. First, our model did not take fatty acid into account in energy imbalance during myocardial I/R injury. We know that oxidation of fatty acid has a competitive relationship with glucose in myocardial energy metabolism [34]. Fatty acid may cause a more complicated, moot result. Second, D-lactate and L-lactate may influence cellular $\mathrm{pH}$ in cardiomyocytes, but no common inhibitors are available.

Our study first showed that MCT1 and MCT4 protein expression are increased during the myocardial I/R period with increases in AMPK phosphorylation. Pharmacological targeting of MCTs may provide new treatment strategies for myocardial ischemia-reperfusion injury. In addition, AMPK may be a potential modulation pathway for MCTs .

\section{Acknowledgements}

This study was supported by grants from the National Natural Science Foundation of China (no.30801076/c160203).

\section{Conflict of Interest}

None.

\section{References}

$>1$ Murray CJ, Lopez AD: Alternative projections of mortality and disability by cause 1990-2020: Global burden of disease study. Lancet 1997;349:1498-1504.

2 Morrison A, Li J: Ppar- $\gamma$ and ampk - advantageous targets for myocardial ischemia/reperfusion therapy. Biochem Pharmacol 2011;82:195-200.

3 Lopaschuk G: Regulation of carbohydrate metabolism in ischemia and reperfusion. Am Heart J 2000;139:S115-119.

4 Vanoverschelde JL, Janier MF, Bakke JE, Marshall DR, Bergmann SR: Rate of glycolysis during ischemia determines extent of ischemic injury and functional recovery after reperfusion. Am J Physiol 1994;267:H1785-1794.

Brooks GA: Lactate: Link between glycolytic and oxidative metabolism. Sports Med 2007;37:341-343. Brooks GA: Cell-cell and intracellular lactate shuttles. J Physiol 2009;587:5591-5600.

Halestrap AP, Wilson MC: The monocarboxylate transporter family-role and regulation. IUBMB Life 2012;64:109-119.

8 Merezhinskaya N, Fishbein WN: Monocarboxylate transporters: Past, present, and future. Histol Histopathol 2009;24:243-264. 


\begin{tabular}{|c|c|c|}
\hline Cellular Physiology & Cell Physiol Biochem 2013;32:663-674 & \\
\hline and Biochemistry & $\begin{array}{l}\text { DOI: 10.1159/000354470 } \\
\text { Published onIIne: September 10, } 2013\end{array}$ & $\begin{array}{l}\text { O) } 2013 \text { S. Karger AG, Basel } \\
\text { www.karger.com/cpb }\end{array}$ \\
\hline
\end{tabular}

-9irk P, Wilson MC, Heddle C, Brown MH, Barclay AN, Halestrap AP: Cd147 is tightly associated with lactate transporters mct1 and mct4 and facilitates their cell surface expression. EMBO J 2000;19:3896-3904.

-10 Brooks GA: Intra- and extra-cellular lactate shuttles. Med Sci Sports Exerc 2000;32:790-799.

11 Galardo MN, Riera MF, Pellizzari EH, Cigorraga SB, Meroni SB: The amp-activated protein kinase activator, 5-aminoimidazole-4-carboxamide-1-b-d-ribonucleoside, regulates lactate production in rat sertoli cells. J Mol Endocrinol 2007;39:279-288.

12 Furugen A, Kobayashi M, Narumi K, Watanabe M, Otake S, Itagaki S, Iseki K: Amp-activated protein kinase regulates the expression of monocarboxylate transporter 4 in skeletal muscle. Life Sciences 2011;88:163168.

13 Kitaoka Y, Machida M, Takemasa T, Hatta H: Expression of monocarboxylate transporter (mct) 1 and mct4 in overloaded mice plantaris muscle. J Physiol Sci 2011;61:467-472.

14 Chin JT, Troke JJ, Kimura N, Itoh S, Wang X, Palmer OP, Robbins RC, Fischbein MP: A novel cardioprotective agent in cardiac transplantation: Metformin activation of amp-activated protein kinase decreases acute ischemia-reperfusion injury and chronic rejection. Yale J Biol Med 2011;84:423-432.

15 Essop MF: Cardiac metabolic adaptations in response to chronic hypoxia. J Physiol 2007;584:715-726.

16 Kantor PF, Dyck JR, Lopaschuk GD: Fatty acid oxidation in the reperfused ischemic heart. Am J Med Sci 1999;318:3-14.

17 Taegtmeyer H: Energy metabolism of the heart: From basic concepts to clinical applications. Curr Probl Cardiol 1994;19:59-113.

18 Taegtmeyer H, Goodwin GW, Doenst T, Frazier OH: Substrate metabolism as a determinant for postischemic functional recovery of the heart. Am J Cardiol 1997;80:3A-10A.

19 Laughlin MR, Taylor J, Chesnick AS, DeGroot M, Balaban RS: Pyruvate and lactate metabolism in the in vivo dog heart. Am J Physiol 1993;264:H2068-2079.

20 McClelland GB, Brooks GA: Changes in mct 1, mct 4, and ldh expression are tissue specific in rats after longterm hypobaric hypoxia. J Appl Physiol 2002;92:1573-1584.

-21 Halestrap AP, Wang X, Poole RC, Jackson VN, Price NT: Lactate transport in heart in relation to myocardial ischemia. Am J Cardiol 1997;80:17A-25A.

22 Halestrap AP: The monocarboxylate transporter family-structure and functional characterization. IUBMB Life 2012;64:1-9.

23 Ullah MS: The plasma membrane lactate transporter mct4, but not mct1, is up-regulated by hypoxia through a hif-1 -dependent mechanism. J Biol Chem 2005;281:9030-9037.

24 Dimmer KS, Friedrich B, Lang F, Deitmer JW, Broer S: The low-affinity monocarboxylate transporter mct4 is adapted to the export of lactate in highly glycolytic cells. Biochem J 2000;350:219-227.

25 Vandenberg JI, Metcalfe JC, Grace AA: Mechanisms of phi recovery after global ischemia in the perfused heart. Circ Res 1993;72:993-1003.

26 Murphy E, Steenbergen C: Ion transport and energetics during cell death and protection. Physiology 2008;23:115-123.

27 Martinov V, Rizvi SMH, Weiseth SA, Sagave J, Bergersen LH, Valen G: Increased expression of monocarboxylate transporter 1 after acute ischemia of isolated, perfused mouse hearts. Life Sci 2009;85:379-385.

28 McNulty PH, Jagasia D, Cline GW, Ng CK, Whiting JM, Garg P, Shulman GI, Soufer R: Persistent changes in myocardial glucose metabolism in vivo during reperfusion of a limited-duration coronary occlusion. Circulation 2000;101:917-922.

29 Chareonthaitawee P, Christian TF, O'Connor MK, Berger PB, Higano ST, O'Keefe JH, Spain MG, Grines CL, Gibbons RJ: Noninvasive prediction of residual blood flow within the risk area during acute myocardial infarction: A multicenter validation study of patients undergoing direct coronary angioplasty. Am Heart J 1997;134:639-646.

30 Matejovic M, Radermacher P, Fontaine E: Lactate in shock: A high-octane fuel for the heart? Intensive Care Med 2007;33:406-408.

31 Chatham JC, Des Rosiers C, Forder JR: Evidence of separate pathways for lactate uptake and release by the perfused rat heart. Am J Physiol Endocrinol Metab 2001;281:E794-802.

-32 Tekkök SB, Brown AM, Westenbroek R, Pellerin L, Ransom BR: Transfer of glycogen-derived lactate from astrocytes to axons via specific monocarboxylate transporters supports mouse optic nerve activity. J Neurosci Res 2005;81:644-652.

-33 Johannsson E, Lunde PK, Heddle C, Sjaastad I, Thomas MJ, Bergersen L, Halestrap AP, Blackstad TW, Ottersen OP, Sejersted OM: Upregulation of the cardiac monocarboxylate transporter mct1 in a rat model of congestive heart failure. Circulation 2001;104:729-734.

34 Awan MM, Saggerson ED: Malonyl-coa metabolism in cardiac myocytes and its relevance to the control of fatty acid oxidation. Biochem J 1993;295:61-66. 